\title{
Recovery of periodicities hidden in heavy-tailed noise
}

\author{
Illya M. Karabash, Jürgen Prestin
}

\begin{abstract}
We address a parametric joint detection-estimation problem for discrete signals of the form $x(t)=\sum_{n=1}^{N} \alpha_{n} \mathrm{e}^{-\mathrm{i} \lambda_{n} t}+\epsilon_{t}, t \in \mathbb{N}$, with an additive noise represented by independent centered complex random variables $\epsilon_{t}$. The distributions of $\epsilon_{t}$ are assumed to be unknown, but satisfying various sets of conditions. We prove that in the case of a heavy-tailed noise it is possible to construct asymptotically strongly consistent estimators for the unknown parameters of the signal, i.e., frequencies $\lambda_{n}$, their number $N$, and complex coefficients $\alpha_{n}$. For example, one of considered classes of noise is the following: $\epsilon_{t}$ are independent identically distributed random variables with $\mathbb{E}\left(\epsilon_{t}\right)=0$ and $\mathbb{E}\left(\left|\epsilon_{t}\right| \ln \left|\epsilon_{t}\right|\right)<\infty$. The construction of estimators is based on detection of singularities of anti-derivatives for $Z$-transforms and on a two-level selection procedure for special discretized versions of superlevel sets. The consistency proof relies on the convergence theory for random Fourier series. We discuss also decaying signals and the case of infinite number of frequencies.
\end{abstract}

Keywords: Random Fourier series, Prony problem, sinusoids in noise, estimation of dimension, asymptotically consistent estimation, consistent localization

MSC-classes: 94A12, 42A70, 42A61, 62F12, 42A24

\section{Contents}

1 Introduction

2 Main results and employed techniques

Acknowledgments. The authors are grateful to the anonymous referee for careful reading of the paper and a number of helpful remarks and questions. The authors would like to thank Frank Filbir, Hrushikesh N. Mhaskar, and Boaz Nadler for valuable discussions. During various parts of this research, the authors were supported by the EU-financed projects AMMODIT ("Approximation Methods for Molecular Modelling and Diagnosis Tools", grant agreement MSCA-RISE-2014-645672AMMODIT) and EUMLS ("EU-Ukrainian Mathematicians for Life Sciences", Marie Curie Actions International Research Staff Exchange Scheme FP7-People-2011-IRSES, project number 295164); the first named author was supported by the Alexander von Humboldt Foundation, by Hausdorff Research Institute for Mathematics, Bonn, and by the project no.15-1vv $\backslash 19$ of Vasyl' Stus Donetsk National University. 
3 Consistent localization of frequencies 5

3.1 Main setting and temporal windows . . . . . . . . . . . . . . 5

3.2 Localization by discrete superlevel arcs . . . . . . . . . . . . . . . 7

3.3 Proof of Theorem $3.2 \ldots \ldots \ldots$

4 Two-threshold estimators for parameters 10

4.1 Almost decreasing anti-derivative kernels . . . . . . . . . . . . . 11

4.2 Strongly consistent estimators of $N, \mathrm{e}^{\mathrm{i} \lambda_{n}}$, and $\alpha_{n} \ldots \ldots \ldots \ldots$

4.3 Proof of Theorem $4.2 \ldots \ldots \ldots \ldots \ldots \ldots$

4.4 Proof of Proposition $4.3 \ldots \ldots \ldots \ldots \ldots$

5 Discussion and additional remarks 18

5.1 Relaxation of signal and noise assumptions . . . . . . . . . . . . . . 18

5.2 Probability estimates for accurate localization . . . . . . . . . . . 19

\section{Introduction}

Consider a signal modeled by the complex time series of the form

$$
x(t)=\sum_{n=1}^{N} \alpha_{n} \mathrm{e}^{-\mathrm{i} \lambda_{n} t}+\epsilon_{t}, \quad t \in \mathbb{N},
$$

consisting of a finite number $N \in \mathbb{Z}^{+}$of periodicities $\alpha_{n} \mathrm{e}^{-\mathrm{i} \lambda_{n} t}$ with distinct real frequencies $\lambda_{n} \in[-\pi, \pi)$ and complex amplitudes $\alpha_{n} \neq 0$. The signal is corrupted by a random noise $\left(\epsilon_{t}\right)_{t=1}^{\infty}$. The problem of finding, or estimation of the unknown parameters $N, \lambda_{n}$, and $\alpha_{n}$ from a finite number of samples $(x(t))_{t=1}^{m}$ is a fundamental problem in signal processing with a number of applications ranging from speech recognition and direction finding in array antennas to astrophysics, medicine, and economics (see [3, 38, 36, 5] and references therein).

When the number $N$ of periodicities is unknown, the number of spectral data is not bounded by a known finite number. So the numerical recovery can be expected only via a convergent process with theoretically infinite number of steps. On each step the process has to use only a finite part of the signal and should give certain approximations to unknown parameters $N, z_{n}:=e^{\mathrm{i} \lambda_{n}}$, and $\alpha_{n}$. These approximations $\widehat{N}=\widehat{N}(m), \widehat{z}_{n}=$ $\widehat{z}_{n}(m), \widehat{\alpha}=\widehat{\alpha}(m)$ are called estimators and, in the case of random noise, are functions of random variables $x(t), t=1, \ldots, m$. An estimator is called (asymptotically) consistent if it converges with $m \rightarrow+\infty$ to the corresponding parameter in a certain probabilistic sense.

This paper is aimed on the case when the random noise variables $\epsilon_{t}$ are allowed to have heavy-tailed distributions and studies strong consistency of estimators, which corresponds to almost sure convergence. The interest to the heavy-tailed $\epsilon_{t}$ is stimulated by the paper of Zhou and Sornette [38], which numerically investigates the case of $\epsilon_{t}$ with infinite variances $\mathbb{E}\left(\left|\epsilon_{t}\right|^{2}\right)$ and lists a number of applications. The latter includes vortices in freely decaying $2-\mathrm{D}$ turbulence, ion-signature precursors of earthquakes, and the price dynamics of speculative bubbles preceding financial collapses. 
The most difficult part of the problem is usually the detection of the unknown number $N$ of periodicities. The presently available analytic proofs of consistency require the assumption $\mathbb{E}\left(\left|\epsilon_{t}\right|^{4}\right)<\infty$. However, the numerical experiments of [38] suggest that consistent estimation is possible even in the case when variances $\mathbb{E}\left(\left|\epsilon_{t}\right|^{2}\right)$ are infinite.

The goal of this paper is to study analytically the case of independent identically distributed (i.i.d.) random variables $\epsilon_{t}$ with infinite variances and to prove that consistent estimation of all unknown parameters is possible under fairly mild assumptions on the distribution tails of $\epsilon_{t}$.

Notation. The following sets of real and complex numbers are used: open halflines $\mathbb{R}_{ \pm}=\{x \in \mathbb{R}: \pm x>0\}$, nonnegative and nonpositive integers $\mathbb{Z}^{ \pm}= \pm \mathbb{N} \cup\{0\}$, the unit circle $\mathbb{T}=\{z \in \mathbb{C}:|z|=1\}$, and the unit disc $\mathbb{D}=\{z \in \mathbb{C}:|z|<1\}$. When we consider the real interval $\left[\theta_{1}, \theta_{2}\right]$, or arcs

$$
\operatorname{Arc}\left[\theta_{1}, \theta_{2}\right]:=\left\{\mathrm{e}^{\mathrm{i} \theta}: \theta_{1} \leq \theta \leq \theta_{2}\right\} \subset \mathbb{T}
$$

we assume that $\theta_{1} \leq \theta_{2}$. For $\zeta \in \mathbb{C}$ and complex sets $G_{1,2}$,

$$
G_{1}+G_{2}:=\left\{z_{1}+z_{2}: z_{1} \in G_{1}, z_{2} \in G_{2}\right\}, \quad \zeta G_{1}:=\left\{\zeta z: z \in G_{1}\right\} .
$$

Let $\mathbb{1}:=\left(u_{t}\right)_{t=1}^{+\infty} \in \mathbb{C}^{\mathbb{N}}$ with $u_{t}=1$ for all $t \in \mathbb{N}$. The notation $\lceil s\rceil$ stands for the ceiling function, i.e., the smallest integer not less than $s ; \ln ^{\delta} s$ is $(\ln s)^{\delta}$. By $\mathcal{L}^{p}$ and $\|\cdot\|_{p}$ we denote the standard Lebesgue spaces of complex-valued functions and the corresponding norms, respectively.

\section{Main results and employed techniques}

The problem of hidden sinusoids has a long history with periodicities in orbital data detected numerically as early as 1754 [3]. The classical statement of the problem involves the discrete signal of form $x(t)=\sum_{n=1}^{N} \alpha_{n} \mathrm{e}^{-\mathrm{i} \lambda_{n} t}$ equidistantly sampled over some finite set of $t$. Initially, the number $N$ of frequencies was supposed to be known and finite. Perhaps, the oldest analytic algorithm dates back to G.R. de Prony (see e.g. [27]). The Prony's method and the essentially equivalent annihilating filter method have a number of advantages. They utilize the minimal possible number of observations, work in the case of damped oscillations, and give exact result in the absence of noise. Besides the assumption that $N$ is given, it is usually assumed that the essential drawback of Prony's method is poor performance when data are too noisy.

For the case of a priori knowledge of a bound on the number $N$ of frequencies, a number of deterministic and statistical methods were developed to deal with signals of type (1.1) involving various models of uncertain or random noise $\epsilon_{t}$. Let us mention various modifications and developments of Prony's and ESPRIT methods [34, 28, 29, [26, 27] (in deterministic settings the ESPRIT method of [25, 34] has some common ideas with Prony's methods, see discussion in [27]), the approach of Jones, Njåstad, and Saff employing Wiener-Levinson filters and Szegö polynomials [9, 23, 22, 21, 2, 5], and a variety of statistical methods [3, 34, 37, 32, 36] (for recent developments on the multivariate case see [12, 30, 14, 15]). 
The problem of determination of the number $N$ of sinusoids attracted attention in mid-80s [3, 36] (it was placed in the list of open problems at the end of [3]). Several statistical methods have been developed to obtain asymptotically consistent estimators for $N$ and the parameters of sinusoids (see [6, 11, 32, 24, 13, 20, 12 and the reviews in [36, 20]).

While most of studies work with a white Gaussian noise with a known or unknown standard deviation, in many practical situations the distribution of noise is unknown. Some steps for lifting of Gaussian and independency assumptions have been done. Kavalieris and Hannan [11] deal with a "colored' autoregressive noise with innovations sequence $\varepsilon(t)$ satisfying $\mathbb{E}\left(\varepsilon(t)^{4}\right)<\infty$ and prove that their estimator provides strongly consistent detection of the number $N$ of sinusoids. The performance of periodogram and information criteria methods in presence of non-Gaussian $\epsilon_{t}$ was studied numerically by Zhou and Sornette [38] and Nadler and Kontorovich [20]. Under the assumption that $N$ is known, the question of probability estimates for localization of $\lambda_{n}$ in the presence of a non-Gaussian noise was raised recently in [5] in connection with a modified method of orthogonal polynomials and the estimation techniques of [18].

Presently there exists a gap between assumptions of analytically proved consistency results and numerical evidences of the fact that many of statistical methods perform well under weaker restrictions on noise.

The main goal of the present paper is to fill this gap and to certify analytically that recovery of the unknown parameters is possible in the case of a heavy-tailed noise. We prove rigorously that strongly consistent estimation of the parameters $N \in \mathbb{Z}^{+}$, $\lambda_{n} \in[-\pi, \pi)$, and $\alpha_{n} \in \mathbb{C} \backslash\{0\}$ of the signal (1.1) is possible in presence of complex random noise sequences $\left(\epsilon_{t}\right)_{t=1}^{\infty}$ belonging to the following classes:

(N1) the random variables $\epsilon_{t}$ are i.i.d. with $\mathbb{E}\left(\epsilon_{t}\right)=0$ and $\mathbb{E}\left(\left|\epsilon_{t}\right| \log \left|\epsilon_{t}\right|\right)<\infty$ for all $t$;

(N2) $\epsilon_{t}$ are i.i.d. symmetric and satisfy $\mathbb{E}\left(\left|\epsilon_{t}\right| \log \log \left(\left|\epsilon_{t}\right|+\mathrm{e}\right)\right)<\infty$.

Besides of heavy-tailed $\epsilon_{t}$, we consider also the case when variances of $\epsilon_{t}$ are finite, but not uniformly bounded in $t$. In this case, the consistency is proved under the assumption of sub-linear growth of $\mathbb{E}\left(\left|\epsilon_{t}\right|^{2}\right)$. More precisely, we will consider the following class of noise sequences:

(N3) $\epsilon_{t}$ are independent, symmetric, and have finite variances $\mathbb{E}\left(\left|\epsilon_{t}\right|^{2}\right)$ satisfying $\mathbb{E}\left(\left|\epsilon_{t}\right|^{2}\right)=O\left(t^{\nu}\right)$ as $t \rightarrow+\infty$ for certain $\nu<1$.

While our approach has some common features with the classical methods (see e.g. [36]) involving detection of peaks of periodograms and Welch temporal windows, it has the following novelties:

- We are aimed at the detection of singularities of the anti-derivative $S_{x}$ of the $Z$-transform of $(x(t))_{t=1}^{+\infty}$. It is easy to see that the anti-derivative of the deterministic signal $y(t)=\sum_{n=1}^{N} \alpha_{n} \mathrm{e}^{-\mathrm{i} \lambda_{n} t}$ defined by

$$
S_{y}\left(\mathrm{e}^{\mathrm{i} \theta}\right):=\sum_{t=1}^{+\infty} \mathrm{e}^{\mathrm{i} t \theta} y(t) / t
$$

has logarithmic singularities at the points $\mathrm{e}^{\mathrm{i} \lambda_{n}}$ of the unit circle $\mathbb{T}=\left\{\mathrm{e}^{\mathrm{i} \theta}: \theta \in \mathbb{R}\right\}$. 
- Strong consistency is proved with the use of uniform convergence theorems for the random series $S_{\epsilon}\left(\mathrm{e}^{\mathrm{i} \theta}\right):=\sum_{t=1}^{+\infty} \mathrm{e}^{\mathrm{i} t \theta} \epsilon_{t} / t$ corresponding to the noise part of $S_{x}$.

This allows us to avoid the estimation of distribution tails of $\mathcal{L}^{\infty}$-norms of random trigonometric polynomials generated by $\epsilon_{t}$, which is not adequately studied in the non-subexponential case. One of the main points of this paper is to show that the results of Kahane [10] and Cuzick and Lai [4] on uniform convergence of random Fourier series are powerful enough for estimation of parameters in the presence of a heavy-tailed noise.

- The estimators for $\lambda_{n}$ are generated not by peaks of partial sums of $S_{x}$, but by discretized versions of superlevel sets associated with special temporal windows, i.e., with partial sums

$$
S_{x, m}^{A}\left(\mathrm{e}^{\mathrm{i} \theta}\right):=\sum_{t=1}^{m} \frac{a_{m, t}}{t} x_{t} \mathrm{e}^{\mathrm{i} t \theta}
$$

defined by a special summation matrix $A=\left(a_{m, t}\right)_{m, t=1}^{+\infty}$, see Sections 3.1 and Proposition 4.1.

- While the type of summation is not important for consistent localization of isolated frequencies (see Section 3.2), it becomes essential for asymptotically consistent detection of frequencies and estimation of their number (Sections 4). Indeed, the noise part $S_{\epsilon}^{A}$ and possible side lobes of summation kernels will produce peaks and superlevel sets of $\left|S_{x}^{A}\right|$ that do not directly correspond to frequencies $\lambda_{n}$, but rather lie nearby and accompany the superlevel sets containing the frequencies.

- To filter out such side superlevel sets we develop in Section 4 a two-level selection approach and employ special kernels with 'almost-monotonicity' property (see Section 4.1).

\section{Consistent localization of frequencies}

\subsection{Main setting and temporal windows}

Consider a discrete signal $x$ of the form (1.1) with a random noise $\left(\epsilon_{t}\right)_{t=1}^{+\infty}$ consisting of independent random variables $\epsilon_{t}$ defined on a complete probability space $(\Omega, \mathcal{A}, \operatorname{Pr})$. We assume that the set $\left\{\lambda_{n}\right\}_{n=1}^{N}$ consists of a finite number $N \in \mathbb{Z}^{+}$of distinct real frequencies $\lambda_{n} \in[-\pi, \pi)$. With the deterministic part $y$ of the signal we associate the complex Borel measure

$$
\mu=\sum_{n=1}^{N} \alpha_{n} \delta\left(z-\mathrm{e}^{\mathrm{i} \lambda_{n}}\right)
$$

on the unit circle $\mathbb{T}$ consisting of (complex) point masses $\alpha_{n} \in \mathbb{C} \backslash\{0\}$ placed at points

$$
z_{n}=\mathrm{e}^{\mathrm{i} \lambda_{n}} .
$$


So every frequency $\lambda_{n}$ corresponds to the $\delta$-function term $\alpha_{n} \delta\left(z-z_{n}\right)$ and the support of the measure is given by

$$
\operatorname{supp} \mu=\left\{z_{n}\right\}_{n=1}^{N} \quad \text { if } N>0 \quad \text { and } \quad \operatorname{supp} \mu=\varnothing \quad \text { if } N=0 .
$$

Then the signal $x$ can be written in the form

$$
x(t)=y(t)+\epsilon_{t}, \quad \text { where } y(t)=\int_{\mathbb{T}} z^{-t} \mathrm{~d} \mu .
$$

The problem under consideration is to develop consistent estimators that recover either the support of $\mu$, or completely the measure $\mu$ from one sample of the random vector $x=(x(t))_{t=1}^{+\infty}=(x(t, \omega))_{t=1}^{+\infty}, \omega \in \Omega$, under certain additional assumptions on random noise components $\epsilon_{t}(\omega)$. Our goal is to construct strongly consistent estimators $\widehat{N}=\widehat{N}(m, \omega), \widehat{z}_{n}=\widehat{z}_{n}(m, \omega)$, and $\widehat{\alpha}_{n}=\widehat{\alpha}_{n}(m, \omega)$, which, by definition, depend only on the finite parts $(x(t))_{t=1}^{m}$ of the signal and have to converge with $m \rightarrow+\infty$ to true spectral data $N, z_{n}$, and $\alpha_{n}$ on a certain almost sure (a.s.) event of the probability space.

With an arbitrary signal $u=(u(t))_{t=1}^{+\infty}$, we associate its weighted modification $W=(u(t) / t)_{t=1}^{+\infty}$ and the geophysical $Z$-transform $S_{u}(z)=\sum_{t=1}^{+\infty} z^{t} u(t) / t$ of $W$ defined as a function of a complex variable $z$ on the set of its convergence. The function $S_{u}$ is an anti-derivative of the $Z$-transform $\sum_{t=0}^{+\infty} z^{t} u(t+1)$ of the shifted signal $(u(t+1))_{t=0}^{+\infty}$.

For the unilateral Fourier series $S_{u}\left(\mathrm{e}^{\mathrm{i} \theta}\right)$ we will use various summation procedures defined via infinite summation matrices $A=\left(a_{m, t}\right)_{m, t=1}^{+\infty}$. Partial sums associated with the matrix $A$ are given by $S_{u, m}^{A}\left(\mathrm{e}^{\mathrm{i} \theta}\right):=\sum_{t=1}^{+\infty} a_{m, t} \frac{u_{t}}{t} \mathrm{e}^{\mathrm{i} t \theta}$.

It will be supposed that $\left(a_{m, t}\right)$ satisfy the following conditions:

$$
\begin{gathered}
0<a_{m, 1} \leq 1 \quad \text { and } a_{m, t} \text { is non-increasing in } t \text { for every } m \in \mathbb{N}, \\
a_{m, t} \geq 0 \text { if } t \leq m, \quad a_{m, t}=0 \text { if } t>m, \\
a_{m, t} \rightarrow 1 \text { as } m \rightarrow+\infty \quad \text { for every } t \in \mathbb{N}, \\
a_{m, t} \text { is non-decreasing in } m \text { for every } t \in \mathbb{N} .
\end{gathered}
$$

Under these conditions $A$-summation is regular. That is, for any strongly convergent series $V_{0}=\sum_{t=1}^{+\infty} V_{t}$ (with $V_{t}$ from a certain Banach space), the sequence of $A$-partial sums $\sum_{t=1}^{m} a_{m, t} V_{t}$ is also convergent to $V_{0}$, which follows from the Toeplitz regularity test, see e.g. [7, Theorem 3.2.2].

We will need the following simple corollary of the regularity of $A$-summation.

Lemma 3.1. Let $\mu$ be a complex Borel measure supported in a finite number of points of $\mathbb{T}$. Then $S_{y, m}^{A}\left(\mathrm{e}^{\mathrm{i} \theta}\right)$ converge uniformly on each closed arc of $\mathbb{T}$ disjoint with $\operatorname{supp} \mu$.

Proof. Note that $S_{y}\left(\mathrm{e}^{\mathrm{i} \theta}\right)=-\sum_{n=1}^{N} \alpha_{n} \log \left(1-\mathrm{e}^{\mathrm{i}\left(\theta-\lambda_{n}\right)}\right) \in \mathcal{L}^{1}(\mathbb{T})$, where the sum converges with respect to the norm of $\mathcal{L}^{1}(\mathbb{T})$. Since $S_{y}\left(\mathrm{e}^{\mathrm{i} \theta}\right)$ is smooth on $\mathbb{T} \backslash \operatorname{supp} \mu$, the partial sums $\sum_{t=1}^{n} \mathrm{e}^{\mathrm{i} t \theta} y(t) / t$ converge uniformly on each closed arc of $\mathbb{T}$ disjoint with supp $\mu$. The regularity of the $A$-summation completes the proof. 
In the next subsection, we will use the notion of set convergence following, e.g., [33. Namely, for $z \in \mathbb{C}, G \subset \mathbb{C}, G_{1,2} \subset \mathbb{C}$, put

$$
\begin{gathered}
\operatorname{dist}(z, G):=\inf _{\zeta \in G}|z-\zeta| \text { if } G \neq \varnothing, \quad \text { and } \operatorname{dist}(z, G)=+\infty \text { if } G=\varnothing \\
\operatorname{dist}_{\mathrm{PH}}\left(G_{1}, G_{2}\right):=\sup _{z \in \mathbb{C}}\left|\operatorname{dist}\left(z, G_{1}\right)-\operatorname{dist}\left(z, G_{2}\right)\right| \text { if } G_{1} \cup G_{2} \neq \varnothing, \\
\text { and } \operatorname{dist}_{\mathrm{PH}}\left(G_{1}, G_{2}\right):=0 \text { if } G_{1}=G_{2}=\varnothing .
\end{gathered}
$$

If both of the sets $G_{1,2}$ are nonempty and closed, dist $t_{\mathrm{PH}}$ is the Pompeiu-Hausdorff distance and can be defined by an alternative formula

$$
\operatorname{dist}_{\mathrm{PH}}\left(G_{1}, G_{2}\right)=\inf \left\{\eta \geq 0: G_{1} \subset G_{2}+\eta \overline{\mathbb{D}}, G_{2} \subset G_{1}+\eta \overline{\mathbb{D}}\right\}
$$

where $\overline{\mathbb{D}}$ is the closed unit disc in $\mathbb{C}$, see [33, formula 4(5)].

Having a sequence $\left(L_{m}\right)_{m=1}^{+\infty}$ of closed subsets of $\mathbb{T}$, we say that $\left(L_{m}\right)_{m=1}^{+\infty}$ converges to a closed set $G$ (and write $\lim L_{m}=G$ ) if $\lim _{m \rightarrow+\infty} \operatorname{dist}_{\mathrm{PH}}\left(L_{m}, G\right)=0$. Since only the subsets of the bounded set $\mathbb{T}$ are considered, this convergence is a restriction of the Painlevé-Kuratowski convergence on closed subsets of $\mathbb{T}$, and coincides with the convergence with respect to Pompeiu-Hausdorff distance whenever $L_{m}$ and $G$ are nonempty.

\subsection{Localization by discrete superlevel arcs}

In this subsection, let us fix an arbitrary summation matrix $A$ satisfying (3.1)-(3.4). The kernel $S_{\mathbb{1}, m}^{A}$ associated with $A$-summation of the anti-derivative is defined by

$$
S_{\mathbb{1}, m}^{A}\left(\mathrm{e}^{\mathrm{i} \theta}\right):=\sum_{t=1}^{m} \frac{a_{m, t}}{t} \mathrm{e}^{\mathrm{i} t \theta} .
$$

The goal of this section is to produce strongly consistent estimators of the support of the measure $\mu$, i.e., to produce random subsets $\widehat{L}_{m}$ of $\mathbb{T}$ that a.s. converge to supp $\mu$ as $m \rightarrow+\infty$. These estimators will have an additional property that, with probability 1 , supp $\mu \subset \widehat{L}_{m}$ for $m$ large enough. This property will be crucial for the construction of estimators for the parameters of the signal in the next section.

The following discrete versions of superlevel sets serve as building blocks for $\widehat{L}_{m}$.

Definition 3.1. We define a discrete superlevel arc of level $h \in \mathbb{R}$ and grid order $J$ for the partial $A$-sum $S_{u, m}^{A}$ (in short, $(h, J)$-arc for $S_{u, m}^{A}$ ) as a closed arc in $\mathbb{T}$ of the form $\operatorname{Arc}\left[\frac{2 \pi j_{1}}{J}, \frac{2 \pi j_{2}}{J}\right]$ with $j_{1,2} \in \mathbb{Z}$ such that

$$
\left|S_{u, m}^{A}\left(\exp \left(\mathrm{i} \frac{2 \pi j}{J}\right)\right)\right| \geq h \quad \text { for all } j \in \mathbb{Z} \cap\left[j_{1}, j_{2}\right] .
$$

Levels and orders of grids will depend on the length $m$ of the signal and have to be connected with the matrix $A$. This is done in the following way. 
Since $S_{\mathbb{1}, m}^{A}(1)=\sum_{t=1}^{m} a_{m, t} / t$ goes to $+\infty$ as $m \rightarrow+\infty$, there exists an auxiliary sequence $\left(H_{m}\right)_{m=1}^{+\infty}$ of real numbers such that

$$
H_{m}<S_{\mathbb{1}, m}^{A}(1) \text { for all } m, \quad \text { and } \lim H_{m}=+\infty .
$$

When such a sequence $\left(H_{m}\right)_{m=1}^{+\infty}$ is chosen, let us fix also a sequence $\left(h_{m}\right)_{m=1}^{+\infty}$ of real numbers satisfying

$$
\lim h_{m}=\lim \frac{H_{m}}{h_{m}}=+\infty,
$$

and a sequence $\left(J_{m}\right)_{m=1}^{+\infty}$ of natural numbers so that

$$
\left|S_{\mathbb{1}, m}^{A}\left(\mathrm{e}^{\mathrm{i} \theta}\right)\right| \geq H_{m} \text { for all } \theta \in\left[-2 \pi / J_{m}, 2 \pi / J_{m}\right] .
$$

The latter is always possible due to the continuity of $S_{\mathbb{1}, m}^{A}\left(\mathrm{e}^{\mathrm{i} \theta}\right)$. Examples will be given below.

Let us note that (3.8), $\lim H_{m}=+\infty$, and Lemma 3.1 imply

$$
\lim J_{m}=+\infty .
$$

For each $m \in \mathbb{N}$, we denote by

$$
\widehat{L}_{m}:=\widehat{L}_{m}(\omega) \text { the union of all }\left(h_{m}, J_{m}\right) \text {-arcs for } S_{x, m}^{A},
$$

which is assumed to be the empty set in the case when such superlevel arcs do not exist.

Theorem 3.2 (consistent localization). Assume that at least one of conditions (N1)(N3) of Section 1 is fulfilled for the random noise $\epsilon$. Then almost surely

the sets $\widehat{L}_{m}$ converge to supp $\mu$ and contain supp $\mu$ for $m$ large enough.

The proof is given in the next subsection. Let us provide an example of possible choices of $H_{m}, h_{m}$, and $J_{m}$ for the case of the Dirichlet summation.

Example 3.3. The matrix $A$ associated with the Dirichlet summation is defined by

$$
a_{m, t}=1 \text { if } t \leq m, \quad a_{m, t}=0 \quad \text { if } t>m .
$$

If $|\theta| \leq \frac{\pi}{4 m}$, one has $\left|S_{\mathbb{1}, m}^{A}\left(\mathrm{e}^{\mathrm{i} \theta}\right)\right| \geq\left|\sum_{t=1}^{m} \frac{\cos (t \theta)}{t}\right| \geq \frac{1}{\sqrt{2}} \sum_{t=1}^{m} \frac{1}{t} \geq \frac{\ln (m+1)}{\sqrt{2}}$. So (3.6), (3.7), and (3.8) are fulfilled if

$$
H_{m}=\frac{\ln (m+1)}{\sqrt{2}}, \quad J_{m}=8 m, \quad \text { and } \quad h_{m}=c_{0}+c_{1} \ln ^{1-\delta} m,
$$

where $\delta \in(0,1), c_{0} \in \mathbb{R}$, and $c_{1} \in \mathbb{R}_{+}$are constants. 


\subsection{Proof of Theorem 3.2}

Recall that $S_{\epsilon}\left(\mathrm{e}^{\mathrm{i} \theta}\right):=\sum_{t=1}^{+\infty} \mathrm{e}^{\mathrm{i} t \theta} \epsilon_{t} / t$ is the random Fourier series associated with the noise part of $S_{x}$.

Proposition 3.4. Assume that $\left\{\epsilon_{t}\right\}_{t=1}^{+\infty}$ satisfies the following condition:

(NO) the set $B_{1}$ of $\omega \in \Omega$ such that $\sup _{m \in \mathbb{N}}\left\|S_{\epsilon, m}^{A}\left(\mathrm{e}^{\mathrm{i} \theta}\right)\right\|_{\infty}<\infty$ is an almost sure event. Then for every $\omega \in B_{1}$ the following statements hold:

(i) each point $z_{n}=\mathrm{e}^{\mathrm{i} \lambda_{n}}$ of $\operatorname{supp} \mu$ is contained in $\widehat{L}_{m}$ for $m$ large enough,

(ii) each closed arc of $\mathbb{T}$ disjoint with $\operatorname{supp} \mu$ is also disjoint with $\widehat{L}_{m}$ for $m$ large enough.

Proof. (i) Let

$$
v(t)=\alpha_{n} \mathrm{e}^{-\mathrm{i} \lambda_{n} t}, \quad t \in \mathbb{N}, \quad \text { and } \quad \mu_{n}:=\mu-\alpha_{n} \delta\left(z-z_{n}\right) .
$$

Then there exists $\gamma>0$ such that $\operatorname{Arc}\left[\lambda_{n}-\gamma, \lambda_{n}+\gamma\right]$ is disjoint with supp $\mu_{n}$. By Lemma [3.1, $S_{y-v, m}^{A}\left(\mathrm{e}^{\mathrm{i} \theta}\right)$ converge uniformly on $\operatorname{Arc}\left[\lambda_{n}-\gamma, \lambda_{n}+\gamma\right]$ as $m \rightarrow+\infty$. This, $\omega \in B_{1}$, and the definition of the a.s. event $B_{1}$ imply

$$
C_{1}:=\sup _{m \in \mathbb{N}} \max _{\left|\theta-\lambda_{n}\right| \leq \gamma}\left|S_{x-v, m}^{A}\left(\mathrm{e}^{\mathrm{i} \theta}\right)\right|<+\infty .
$$

It follows from $\lim J_{m}=+\infty$ that there exists $m_{1}$ such that $2 \pi / J_{m} \leq \gamma$ for $m \geq m_{1}$. For such $m$, formulas (3.8) and (3.6) imply for $\theta$ satisfying $\left|\theta-\lambda_{n}\right| \leq 2 \pi / J_{m}$ that

$$
\left|S_{x, m}^{A}\left(\mathrm{e}^{\mathrm{i} \theta}\right)\right| \geq\left|S_{v, m}^{A}\left(\mathrm{e}^{\mathrm{i} \theta}\right)\right|-\left|S_{x-v, m}^{A}\left(\mathrm{e}^{\mathrm{i} \theta}\right)\right| \geq\left|\alpha_{n} S_{\mathbb{1}, m}^{A}\left(\mathrm{e}^{\mathrm{i}\left(\theta-\lambda_{n}\right)}\right)\right|-C_{1} \geq\left|\alpha_{n}\right| H_{m}-C_{1} .
$$

By (3.6) and (3.7), there exists $m_{2} \geq m_{1}$ such that $\left|S_{x, m}^{A}\left(\mathrm{e}^{\mathrm{i} \theta}\right)\right| \geq h_{m}$ for $m \geq m_{2}$ and $\left|\theta-\lambda_{n}\right| \leq 2 \pi / J_{m}$.

Thus, for $m \geq m_{2}$, the point $\mathrm{e}^{\mathrm{i} \lambda_{n}}$ is contained in one of $\left(h_{m}, J_{m}\right)$-arcs for $S_{x, m}^{A}$, and so $\mathrm{e}^{\mathrm{i} \lambda_{n}}$ is contained in $\widehat{L}_{m}$.

(ii) Consider an $\operatorname{arc} \operatorname{Arc}\left[\theta_{1}, \theta_{2}\right]$ disjoint with $\operatorname{supp} \mu$. By Lemma 3.1 and the definition of the event $B_{1}$,

$$
C_{2}:=\sup _{m \in \mathbb{N}} \max _{\theta_{1} \leq \theta \leq \theta_{2}}\left|S_{x, m}^{A}\left(\mathrm{e}^{\mathrm{i} \theta}\right)\right|<\infty .
$$

This implies that $\operatorname{Arc}\left[\theta_{1}, \theta_{2}\right]$ is disjoint with $\widehat{L}_{m}$ as soon as $C_{2}<h_{m}$. Condition (3.7) completes the proof.

Consider one more noise class defined by the following assumptions:

(N4) $\epsilon_{t}$ are independent, symmetric, and have finite variances $\mathbb{E}\left(\left|\epsilon_{t}\right|^{2}\right)$ satisfying the condition $\sum_{k=1}^{+\infty} \tau_{k}<+\infty$, where $\tau_{k}:=2^{k / 2}\left(\sum_{t=2^{2^{k}}}^{-1+2^{2^{(k+1)}}} \frac{\mathbb{E}\left(\left|\epsilon_{t+1}\right|^{2}\right)}{(t+1)^{2}}\right)^{1 / 2}$.

Lemma 3.5. Each of conditions (N1)-(N4) implies (NO). 
Proof. The implications $(\mathrm{N} 1) \Rightarrow(\mathrm{N} 0)$ and $(\mathrm{N} 2) \Rightarrow(\mathrm{N} 0)$ follow from [4]. Indeed, 4] implies that, for each of the classes (N1) and (N2), usual partial sums $\sum_{t=1}^{m} \epsilon_{t} \mathrm{e}^{\mathrm{i} t \theta} / t$ a.s. converge uniformly on $\mathbb{T}$. The regularity of the $A$-summation process implies that $S_{\epsilon, m}^{A}$ a.s. converge uniformly on $\mathbb{T}$, and so are a.s. uniformly bounded.

Similarly, (N4) $\Rightarrow(\mathrm{N} 0)$ follows from the results of [10, Section 7.2].

To show $(\mathrm{N} 3) \Rightarrow(\mathrm{N} 4)$, it is enough to notice that, under the assumptions $\nu \in(0,1)$ and $\mathbb{E}\left(\left|\epsilon_{t}\right|^{2}\right)<C t^{\nu}$, we have

$$
\begin{aligned}
\tau_{k} \leq & C^{1 / 2} 2^{k / 2}\left(\sum_{t=2^{2^{k}}}^{-1+2^{2^{(k+1)}}}(t+1)^{(\nu-2)}\right)^{1 / 2} \\
& \leq C^{1 / 2}(1-\nu)^{1 / 2} 2^{k / 2}\left(2^{(\nu-1) 2^{k}}-2^{(\nu-1) 2^{(k+1)}}\right)^{1 / 2} \leq C^{1 / 2}(1-\nu)^{1 / 2} 2^{k / 2} 2^{(\nu-1) 2^{k-1}}
\end{aligned}
$$

and so $\sum_{k=1}^{+\infty} \tau_{k}<+\infty$.

Now, Theorem 3.2 easily follows from (3.5), Proposition 3.4, and Lemma 3.5.

Remark 3.1. The above proof shows that condition (N3) can be seen as a transparent particular case of the cumbersome looking condition (N4). We do not expect that under assumption of i.i.d. and $\mathbb{E}\left(\left|\epsilon_{t}\right|^{2}\right)<\infty$ the estimators developed below in Theorem 4.2 are more computationally efficient than that of [20, 38]. The main point of Theorem 4.2 and conditions (N1)-(N3) is to certify analytically that consistent estimation is possible for a non-Gaussian noise and, in this sense, to support numerical experiments of [20, 38].

\section{Two-threshold estimators for parameters}

The estimators $\widehat{L}_{m}$ of $\operatorname{supp} \mu$ constructed in the previous section consist of a finite number of maximal superlevel $\left(h_{m}, J_{m}\right)$-arcs. We will say that a maximal $\left(h_{m}, J_{m}\right)$-arc is a localization arc if it contains at least one point of supp $\mu$.

Assuming that an element $\omega \in \Omega$ belongs to the almost sure event $B_{1}$ of Proposition 3.4, we see that for large enough $m \geq M_{0}=M_{0}(\omega)$ every localization arc contains exactly one point of $\operatorname{supp} \mu$. Hence, the number of localization arcs is a strongly consistent estimator of the number $N$ of frequencies. Since localization arcs converge to one-point sets $\left\{\mathrm{e}^{\mathrm{i} \lambda_{n}}\right\}$ as $m \rightarrow+\infty$, the middle point of the localization arcs are strongly consistent estimator of $z_{n}$.

The problem arising in realization of this approach is possible presence of maximal $\left(h_{m}, J_{m}\right)$-arcs that do not contain any points of $\operatorname{supp} \mu$. We will call them side arcs. The convergence $\widehat{L}_{m} \rightarrow \operatorname{supp} \mu$ implies that, with growth of $m$, side arcs have to lie in smaller and smaller neighborhoods of points $\mathrm{e}^{\mathrm{i} \lambda_{n}}$ and so in smaller and smaller neighborhoods of associated localization arcs.

The goal of this section is to provide a method that filters out side arcs, and so, detects localization arcs. This will lead to a construction of strongly consistent estimators of all parameters. 
The filtering will require a special type of summation process and an additional level sequence $\left(h_{m}^{\prime}\right)_{m=1}^{+\infty}$ of real numbers satisfying

$$
h_{m} \leq h_{m}^{\prime} \quad \text { and } \quad \lim \left(h_{m}^{\prime}-h_{m}\right)=\lim \frac{H_{m}}{h_{m}^{\prime}}=+\infty .
$$

\subsection{Almost decreasing anti-derivative kernels}

There can be two causes of appearance of side arcs: (i) random fluctuations of $S_{x, m}^{A}\left(\mathrm{e}^{\mathrm{i} \theta}\right)$ due to the noise component $S_{\epsilon, m}^{A}$, and (ii) spectral leakage arising because of sidelobes (side peaks) of the anti-derivative kernel $\left|S_{\mathbb{1}, m}^{A}\left(\mathrm{e}^{\mathrm{i} \theta}\right)\right|$.

To eliminate the second effect, we will use kernels with a special property, which provide bounds uniform in $m$ on fluctuations from the main lobe. Namely, additionally to assumptions (3.1)-(3.4), we will suppose that the kernel $S_{\mathbb{1}, n}^{A}\left(\mathrm{e}^{\mathrm{i} \theta}\right)$ is almost decreasing in the following sense: for each $m \in \mathbb{N}$,

$$
S_{\mathbb{1}, m}^{A}\left(\mathrm{e}^{\mathrm{i} \theta}\right)=f_{m}(\theta)+\mathfrak{b}(m, \theta),
$$

where $f_{m}(\theta)$ is a $2 \pi$-periodic even nonnegative function non-increasing on $[0, \pi]$,

$$
\mathfrak{b}(m, \cdot) \in \mathcal{L}^{\infty}(\mathbb{R}) \text { and satisfy }|\mathfrak{b}|_{\infty}:=\sup _{m \in \mathbb{N}}\|\mathfrak{b}(m, \theta)\|_{\infty}<\infty
$$

An example of such a summation matrix is given by the following proposition.

Proposition 4.1. For a fixed constant $C>0$, let us consider a sequence $r_{m}=\mathrm{e}^{-C / m}$, $m \in \mathbb{N}$, and the truncated Poisson summation matrix $A$ defined by

$$
a_{m, t}=r_{m}^{t} \quad \text { if } t \leq m, \quad a_{m, t}=0 \quad \text { if } t>m .
$$

Then

$$
\left|S_{\mathbb{1}, m}^{A}\left(\mathrm{e}^{\mathrm{i} \theta}\right)+\ln \left(1-r_{m} \mathrm{e}^{\mathrm{i} \theta}\right)\right|<\int_{C}^{+\infty} \frac{\mathrm{e}^{-x}}{x} \mathrm{~d} x, \quad m \in \mathbb{N},
$$

and the matrix $A$ satisfies (4.2)-(4.4).

Proof. To prove (4.6) it is enough to notice that

$$
\left|S_{\mathbb{1}, m}^{A}\left(\mathrm{e}^{\mathrm{i} \theta}\right)+\ln \left(1-r_{m} \mathrm{e}^{\mathrm{i} \theta}\right)\right| \leq \sum_{t=m+1}^{+\infty} \frac{r_{m}^{t}}{t}<\int_{m}^{+\infty} \frac{\mathrm{e}^{-C x / m}}{x} \mathrm{~d} x .
$$

Then properties (4.2)-(4.4) easily follow from (4.6).

It is obvious that $A$ defined by (4.5) satisfies (3.1)-(3.4). 


\subsection{Strongly consistent estimators of $N, \mathrm{e}^{\mathrm{i} \lambda_{n}}$, and $\alpha_{n}$}

In this subsection we assume that the matrix $A$ satisfies (3.1)-(3.3) and the almost decreasing property (4.2)-(4.4). We assume also that the sequences of $H_{m}, h_{m}, h_{m}^{\prime}$, and $J_{m}$ are chosen in accordance with (3.6), (3.7), (4.1), and (3.8).

We will say that an $(h, J)$-arc $\mathfrak{A}$ for $S_{u, m}^{A}$ is maximal if it is not a subset of any other $(h, J)$-arc for $S_{u, m}^{A}$. This means that either $\mathfrak{A}$ contains all points of the form $\exp \left(\mathrm{i} \frac{2 \pi j}{J}\right)$, $j \in \mathbb{Z}$, or $\mathfrak{A}=\operatorname{Arc}\left[\frac{2 \pi j_{1}}{J}, \frac{2 \pi j_{2}}{J}\right]$ with $j_{1,2} \in \mathbb{Z}$ such that

$$
\begin{aligned}
\left|S_{u, m}^{A}\left(\exp \left(\mathrm{i} \frac{2 \pi\left(j_{1}-1\right)}{J}\right)\right)\right| & <h, \quad\left|S_{u, m}^{A}\left(\exp \left(\mathrm{i} \frac{2 \pi\left(j_{2}+1\right)}{J}\right)\right)\right|<h, \\
& \text { and }\left|S_{u, m}^{A}\left(\exp \left(\mathrm{i} \frac{2 \pi j}{J}\right)\right)\right| \geq h \text { for all } j \in \mathbb{Z} \cap\left[j_{1}, j_{2}\right] .
\end{aligned}
$$

Definition 4.1. By $\mathbb{A}_{m}(u)$ we denote the family of all maximal $\left(h_{m}, J_{m}\right)$-arcs for $S_{u, m}^{A}$ that contain at least one $\left(h_{m}^{\prime}, J_{m}\right)$-arc for $S_{u, m}^{A}$. By $\widehat{N}_{m}(u)$ we denote the number of such maximal arcs in the family $\mathbb{A}_{m}(u)$. If $\mathbb{A}_{m}(u)$ is empty, $\widehat{N}_{m}(u):=0$.

For the case when $u$ is the random signal $x=(x(t, \omega))_{t=1}^{+\infty}$, we will use the shortenings $\mathbb{A}_{m}=\mathbb{A}_{m}(x)$ and $\widehat{N}_{m}=\widehat{N}_{m}(x)$. The following theorem states, in particular, that for large $m$, the random family $\mathbb{A}_{m}$ coincides almost surely with the family of localization arcs, and that $\widehat{N}_{m}$ is a strongly consistent estimator of $N$.

Theorem 4.2. Assume that at least one of the noise conditions (N1)-(N4) of Sections 1 and 3.3 is fulfilled, and consequently, by Lemma 3.5, the $\omega$-set $B_{1}$ defined in condition (NO) is an almost sure event.

Then for $\omega \in B_{1}$ the following statements hold:

(i) There exists $M=M(\omega) \in \mathbb{N}$ such that for all $m \geq M$,

$$
\widehat{N}_{m}=N
$$

and, for each $n \in \mathbb{N}$ so that $1 \leq n \leq N$,

$$
\text { there exists a maximal }\left(h_{m}, J_{m}\right) \text {-arc } \mathfrak{A}_{m, n} \in \mathbb{A}_{m} \text { satisfying } \mathrm{e}^{\mathrm{i} \lambda_{n}} \in \mathfrak{A}_{m, n} .
$$

This means that for $m \geq M$, the family $\mathbb{A}_{m}$ consists exactly of $N$ maximal superlevel arcs $\mathfrak{A}_{m, 1}, \ldots, \mathfrak{A}_{m, N}$. In the case $N=0$, the family $\mathbb{A}_{m}$ is empty.

(ii) Suppose $N \neq 0$ and $m \geq M$. For each $n=1, \ldots, N$, denote by $j_{m, n}$ and $j_{m, n}^{\prime}$ the natural numbers such that $\mathfrak{A}_{m, n}=\operatorname{Arc}\left[2 \pi \mathrm{i} \frac{j_{m, n}}{J_{m}}, 2 \pi \mathrm{i} \frac{j_{m, n}^{\prime}}{J_{m}}\right]$. Then

$$
\widehat{z}_{m, n}:=\exp \left(2 \pi \mathrm{i} \frac{j_{m, n}+j_{m, n}^{\prime}}{2 J_{m}}\right) \quad \text { converge to } z_{n}=\mathrm{e}^{\mathrm{i} \lambda_{n}} \quad \text { as } m \rightarrow+\infty \text {. }
$$

If, additionally to (3.8), the sequence $\left(J_{m}\right)_{m=1}^{+\infty}$ satisfies

$$
\lim _{m \rightarrow+\infty} \frac{S_{\mathbb{1}, m}^{A}\left(\mathrm{e}^{2 \pi \mathrm{i} / J_{m}}\right)}{S_{\mathbb{1}, m}^{A}(1)}=1,
$$


and $j_{m, n}^{\max } \in\left[j_{m, n}, j_{m, n}^{\prime}\right] \cap \mathbb{Z}$ is a maximizer in the sense

$$
\left|S_{x, m}^{A}\left(\exp \left(2 \pi \mathrm{i} \frac{j_{m, n}^{\max }}{J_{m}}\right)\right)\right|=\max _{j_{m, n} \leq j \leq j_{m, n}^{\prime}}\left|S_{x, m}^{A}\left(\exp \left(2 \pi \mathrm{i} \frac{j}{J_{m}}\right)\right)\right|,
$$

then

$$
\alpha_{n}=\lim _{m \rightarrow+\infty} \frac{S_{x, m}^{A}\left(\exp \left(2 \pi \mathrm{i} \frac{j_{m, n}^{\max }}{J_{m}}\right)\right)}{\sum_{t=1}^{m} a_{m, t} / t}
$$

The proof is given in the next subsection. The existence of $\left(h_{m}^{\prime}\right)_{m=1}^{+\infty}$ and $\left(J_{m}\right)_{m=1}^{+\infty}$ satisfying (4.1) and (4.8) is obvious. A particular example is given by the next statement.

Proposition 4.3. Let $A$ be the truncated Poisson summation matrix defined by

$$
a_{m, t}=\mathrm{e}^{-t / m} \text { if } t \leq m, \quad a_{m, t}=0 \text { if } t>m .
$$

Then conditions (3.6), (3.7), (3.8), and (4.1) are fulfilled if $H_{m}, h_{m}, h_{m}^{\prime}$ are defined by

$$
H_{m}=\frac{1}{2} \ln (m)-\frac{1}{2}, \quad h_{m}=c_{3} \ln ^{1-\delta}(m)+c_{4}, \quad h_{m}^{\prime}=c_{5} \ln ^{1-\delta}(m)+c_{6}
$$

where $\delta \in(0,1)$ and $c_{3,4,5,6} \in \mathbb{R}$ are arbitrary constants such that $0<c_{3}<c_{5}, c_{4}<c_{6}$, and $J_{m}$ satisfy $J_{m} \geq 2 \pi \sqrt{m}$.

If, additionally,

$$
J_{m} \geq 2 \pi m^{3 / 2}
$$

then (4.8) is also fulfilled.

The proof is given in Section 4.4 .

Remark 4.1. It is clear that the following statements involving a maximal $\left(h_{m}, J_{m}\right)$ arc $\mathfrak{A}$ for $S_{u, m}^{A}$ are equivalent: (i) $\mathfrak{A}$ contains at least one $\left(h_{m}^{\prime}, J_{m}\right)$-arc, (ii) $\mathfrak{A}$ contains at least one maximal $\left(h_{m}^{\prime}, J_{m}\right)$-arc, (iii) $\mathfrak{A}$ contains a point $z_{0}$ of form $z_{0}=\exp \left(2 \pi \mathrm{i} \frac{j}{J_{m}}\right)$ with $j \in \mathbb{Z}$ such that $\left|S_{u, m}^{A}\left(z_{0}\right)\right| \geq h_{m}^{\prime}$.

Remark 4.2. For large $m$, the sets $\widehat{L}_{m}^{\prime}$ defined as the unions of all $\left(h_{m}^{\prime}, J_{m}\right)$-arcs for $S_{x, m}^{A}$ obviously provide a better localization for $\operatorname{supp} \mu$ than $\widehat{L}_{m}$. Namely, for $\omega \in B_{1}$,

$$
\mathrm{e}^{\mathrm{i} \lambda_{n}} \in \widehat{L}_{m}^{\prime} \cap \mathfrak{A}_{m, n} \text { for large enough } m, \quad \text { and } \quad\left\{\mathrm{e}^{\mathrm{i} \lambda_{n}}\right\}=\lim _{m \rightarrow+\infty} \widehat{L}_{m}^{\prime} \cap \mathfrak{A}_{m, n} .
$$

Since in the settings of (4.9), $\exp \left(2 \pi \mathrm{i} \frac{j_{m, n}^{\max }}{J_{m}}\right) \in \mathfrak{A}_{m, n} \cap \widehat{L}_{m}^{\prime}$, the procedure of finding of $\max _{j_{m, n} \leq j \leq j_{m, n}^{\prime}}\left|S_{x, m}^{A}\left(\exp \left(2 \pi \mathrm{i} \frac{j}{J_{m}}\right)\right)\right|$ can be restricted to the numbers $\exp \left(2 \pi \mathrm{i} \frac{j}{J_{m}}\right)$ lying in the more narrow set $\mathfrak{A}_{m, n} \cap \widehat{L}_{m}^{\prime}$. 
Assume that a finite signal $\{x(t)\}_{t=1}^{M}$ of a sufficiently large length $M$ is given and, for $m \leq M$, the summation matrix $\left(a_{m, t}\right)$, the levels $h_{m}, h_{m}^{\prime}$, and the frequency grid orders $J_{m}$ are prescribed in accordance with (4.11)-(4.13). If $m$ is large enough to ensure that at least one of the numbers $\left|S_{x, m}^{A}\left(\exp \left(2 \pi \mathrm{i} \frac{j}{J_{m}}\right)\right)\right|, j=1, \ldots, J_{m}$, is less than the level $h_{m}$, then the algorithmic summary for computation of the estimators of $z_{n}$ and $\alpha_{n}, n=1, \ldots, N$, by first $m$ values of $x$ is as follows.

\section{Algorithm 4.1.}

Input: $m, J_{m} \in \mathbb{N}, x(t) \in \mathbb{C}$ and $a_{m, t} \in(0,1](t=1, \ldots, m), h_{m}, h_{m}^{\prime} \in \mathbb{R}_{+}$.

Step 1. Compute $S_{x, m}^{A}\left(\mathrm{e}^{2 \pi \mathrm{i} j / J_{m}}\right)$ for $j=1, \ldots, J_{m}$ by formula (2.1).

Step 2. Find all $j \in\left[1, J_{m}\right] \cap \mathbb{N}$ such that $\left|S_{x, m}^{A}\left(\mathrm{e}^{2 \pi \mathrm{i} j / J_{m}}\right)\right| \geq h_{m}$.

Step 3. Find all maximal $\left(h_{m}, J_{m}\right)$-arcs using Definition 3.1.

Step 4. Find the family $\mathbb{A}_{m}(x)=\left\{\mathfrak{A}_{m, n}\right\}_{n=1}^{\widehat{N}_{m}}\left(\widehat{N}_{m} \in \mathbb{Z}^{+}\right)$of all maximal $\left(h_{m}, J_{m}\right)$-arcs $\mathfrak{A}_{m, n}=\operatorname{Arc}\left[2 \pi \mathrm{i} \frac{j_{m, n}}{J_{m}}, 2 \pi \mathrm{i} \frac{j_{m, n}^{\prime}}{J_{m}}\right]\left(j_{m, n}, j_{m, n}^{\prime} \in \mathbb{N}\right)$ that, according to Definition 4.1, contain a point $\zeta_{n}$ of form $\zeta_{n}=\exp \left(2 \pi \mathrm{i} \frac{j}{J_{m}}\right), j \in\left[j_{m, n}, j_{m, n}^{\prime}\right] \cap \mathbb{N}$, satisfying $\left|S_{u, m}^{A}\left(\zeta_{n}\right)\right| \geq h_{m}^{\prime}$.

Output: the estimator $\widehat{N}_{m}$ of the number of frequencies.

Step 5. If $\widehat{N}_{m}=0$, then stop.

Otherwise (when $\widehat{N}_{m} \geq 1$ ) continue with the following steps:

Step 6. For $n=1, \ldots, \widehat{N}_{m}$, compute the estimator $\widehat{z}_{m, n}:=\exp \left(2 \pi \mathrm{i} \frac{j_{m, n}+j_{m, n}^{\prime}}{2 J_{m}}\right)$.

Step 7. For $n=1, \ldots, \widehat{N}_{m}$, compute the estimator $\widehat{\alpha}_{m, n}$ by the averaging formula $\widehat{\alpha}_{m, n}=\frac{1}{I \sum_{t=1}^{m} a_{m, t} / t} \sum_{i=1}^{I} S_{x, m}^{A}\left(\exp \left(2 \pi \mathrm{i} \frac{j_{m, n}^{\max , i}}{J_{m}}\right)\right)$, where $\left\{j_{m, n}^{\max , i}\right\}_{i=1}^{I}$ is the set $\underset{j \in\left[j_{m, n}, j_{m, n}^{\prime}\right] \cap \mathbb{Z}}{\arg \max }\left|S_{x, m}^{A}\left(\exp \left(2 \pi \mathrm{i} \frac{j}{J_{m}}\right)\right)\right|$ of maximizers in the sense of (44.9) , and $I \in \mathbb{N}$ is their number.

Output: $\widehat{z}_{m, n}, \widehat{\alpha}_{m, n}, n=1, \ldots, \widehat{N}$.

Remark 4.3. Actually, by the proof of Theorem 4.2 , any rule choosing a point $\widehat{z}_{m, n} \in$ $\mathfrak{A}_{m, n}$ provides consistent estimators $\widehat{z}_{m, n}$. The formula $\widehat{z}_{m, n}=\exp \left(2 \pi \mathrm{i} \frac{j_{m, n}+j_{m, n}^{\prime}}{2 J_{m}}\right)$ was chosen only because of its simplicity. A more practical choice may involve the number $j_{m, n}^{\max } \in\left[j_{m, n}, j_{m, n}^{\prime}\right] \cap \mathbb{N}$ that is a maximizer in the sense of (4.9)), or the average of such maximizers in a very improbable case of several of them.

\subsection{Proof of Theorem 4.2}

When $N=0$, Theorem 4.2 follows from Proposition 3.4 (ii). Consider the case $N>0$. Let us fix natural $n \leq N$ and define the sequence $(v(t))_{t=1}^{+\infty}$ and the measure $\mu_{n}$ by (3.13).

Statement (i) of Theorem 4.2 follows from Proposition 3.4 and the next proposition. 
Proposition 4.4. Let $\operatorname{Arc}\left[\lambda_{n}-\gamma, \lambda_{n}+\gamma\right]$ with a certain $\gamma>0$ be disjoint with the support of the measure $\mu_{n}$, and let $\omega \in B_{1}$. Then:

(a) There exists a natural number $M_{n}=M_{n}(\gamma, \omega)$ such that for $m \geq M_{n}$ the following statements hold:

(i) in the family $\mathbb{A}_{m}$ there exists exactly one maximal $\left(h_{m}, J_{m}\right)$-arc $\mathfrak{A}_{m, n}$ that has a nonempty intersection with $\operatorname{Arc}\left[\lambda_{n}-\gamma, \lambda_{n}+\gamma\right]$,

(ii) $\mathfrak{A}_{m, n} \subset \operatorname{Arc}\left[\lambda_{n}-\gamma, \lambda_{n}+\gamma\right]$,

(iii) $\mathrm{e}^{\mathrm{i} \lambda_{n}} \in \mathfrak{A}_{m, n}$.

(b) As $m \rightarrow+\infty, \mathfrak{A}_{m, n}$ converge to the one-point set $\left\{\mathrm{e}^{\mathrm{i} \lambda_{n}}\right\}$.

Proof. Proposition 3.4 and Remark 4.1 applied to $\left(h_{m}, J_{m}\right)$-arcs and to $\left(h_{m}^{\prime}, J_{m}\right)$-arcs imply the existence of a number $m_{3}$ such that for all $m \geq m_{3}$ there exist a maximal $\left(h_{m}^{\prime}, J_{m}\right)$-arc $\mathfrak{A}_{m, n}^{\prime}$ and a maximal $\left(h_{m}, J_{m}\right)$-arc $\mathfrak{A}_{m, n}$ such that

$$
\mathrm{e}^{\mathrm{i} \lambda_{n}} \in \mathfrak{A}_{m, n}^{\prime} \subset \mathfrak{A}_{m, n} \subset \operatorname{Arc}\left[\lambda_{n}-\gamma+\frac{2 \pi}{J_{m}}, \lambda_{n}+\gamma-\frac{2 \pi}{J_{m}}\right] .
$$

These inclusions imply $\mathfrak{A}_{m, n} \in \mathbb{A}_{m}$ and the statements (a.ii)-(a.iii).

To prove (a.i), assume that $m \geq m_{3}$, and that there exists

$$
\mathrm{e}^{\mathrm{i} \theta_{0}} \in \operatorname{Arc}\left[\lambda_{n}-\gamma, \lambda_{n}+\gamma\right] \backslash \mathfrak{A}_{m, n} \text { so that } \theta_{0} \text { is of the form } \frac{2 \pi j}{J_{m}}, \quad j \in \mathbb{Z} \text {. }
$$

Then

$$
\left|S_{x, m}^{A}\left(\mathrm{e}^{\mathrm{i} \theta_{0}}\right)\right| \leq\left|\alpha_{n} S_{\mathbb{1}, m}^{A}\left(\mathrm{e}^{\mathrm{i}\left(\theta_{0}-\lambda_{n}\right)}\right)\right|+\left|S_{x-v, m}^{A}\left(\mathrm{e}^{\mathrm{i} \theta_{0}}\right)\right| \leq\left|\alpha_{n}\right|\left(f_{m}\left(\theta_{0}-\lambda_{n}\right)+|\mathfrak{b}|_{\infty}\right)+C_{1},
$$

where $C_{1}$ is the finite number defined by (3.14).

Since $\mathrm{e}^{\mathrm{i} \lambda_{n}} \in \mathfrak{A}_{m, n}, \mathrm{e}^{\mathrm{i} \theta_{0}} \notin \mathfrak{A}_{m, n}$, and $\mathfrak{A}_{m, n}$ is a maximal $\left(h_{m}, J_{m}\right)$-arc, one can see that the subarc of Arc $\left[\lambda_{n}-\gamma, \lambda_{n}+\gamma\right]$ connecting $\mathrm{e}^{\mathrm{i} \lambda_{n}}$ and $\mathrm{e}^{\mathrm{i} \theta_{0}}$ contains a point $\mathrm{e}^{\mathrm{i} \theta_{1}}$ such that

$$
\left|S_{x, m}^{A}\left(\mathrm{e}^{\mathrm{i} \theta_{1}}\right)\right|<h_{m} .
$$

Since $f_{m}$ is monotone on $[-\pi, 0]$ and $[0, \pi]$, we see that

$$
f_{m}\left(\theta_{0}-\lambda_{n}\right) \leq f_{m}\left(\theta_{1}-\lambda_{n}\right)
$$

where, if necessary, a multiple of $2 \pi$ is added to $\theta_{0,1}$ to ensure $\left|\theta_{i}-\lambda_{n}\right| \leq \pi, i=0,1$. On the other side,

$$
f_{m}\left(\theta_{1}-\lambda_{n}\right) \leq\left|S_{\mathbb{1}, m}^{A}\left(\mathrm{e}^{\mathrm{i}\left(\theta_{1}-\lambda_{n}\right)}\right)\right|+|\mathfrak{b}|_{\infty}=\left|\alpha_{n}\right|^{-1}\left|S_{v, m}^{A}\left(\mathrm{e}^{\mathrm{i} \theta_{1}}\right)\right|+|\mathfrak{b}|_{\infty}
$$

and

$$
\left|S_{v, m}^{A}\left(\mathrm{e}^{\mathrm{i} \theta_{1}}\right)\right| \leq\left|S_{x, m}^{A}\left(\mathrm{e}^{\mathrm{i} \theta_{1}}\right)\right|+C_{1} .
$$

Combining these inequalities with (4.17) and (4.18), we see that

$$
f_{m}\left(\theta_{0}-\lambda_{n}\right) \leq\left|\alpha_{n}\right|^{-1}\left(h_{m}+C_{1}\right)+|\mathfrak{b}|_{\infty}
$$


The latter and (4.16) imply

$$
\left|S_{x, m}^{A}\left(\mathrm{e}^{\mathrm{i} \theta_{0}}\right)\right| \leq h_{m}+2 C_{1}+2|\mathfrak{b}|_{\infty}\left|\alpha_{n}\right|
$$

By (4.1), there exists $m_{4} \geq m_{3}$ such that $h_{m}^{\prime}-h_{m}>2 C_{1}+2|\mathfrak{b}|_{\infty}\left|\alpha_{n}\right|$ for $m \geq m_{4}$. For such $m$ we have $\left|S_{x, m}^{A}\left(\mathrm{e}^{\mathrm{i} \theta_{0}}\right)\right|<h_{m}^{\prime}$. Since the last inequality is valid for any $\mathrm{e}^{\mathrm{i} \theta_{0}}$ satisfying (4.15), we see that for $m \geq m_{4}$ there are no $\left(h_{m}^{\prime}, J_{m}\right)$-arcs lying outside $\mathfrak{A}_{m, n}$ and intersecting $\operatorname{Arc}\left[\lambda_{n}-\gamma, \lambda_{n}+\gamma\right]$. Thus, (a.i) is proved.

The statement (b) follows from (a.i) and (4.14). This completes the proof.

Let us prove statement (ii) of Theorem 4.2. The convergence of (4.7) is obvious from Proposition 4.4. Now, we assume (4.8) and prove (4.10).

Let us denote $\theta_{m, j}:=2 \pi \frac{j}{J_{m}}$ for $j \in\left[j_{m, n}, j_{m, n}^{\prime}\right] \cap \mathbb{Z}$ and $\theta_{m}^{\max }:=2 \pi \frac{j_{m, n}^{\max }}{J_{m}}$. Note that

$$
\sum_{t=1}^{m} a_{m, t} / t=S_{\mathbb{1}, m}^{A}(1)=\left\|S_{\mathbb{1}, m}^{A}\left(\mathrm{e}^{\mathrm{i} \theta}\right)\right\|_{\infty}=\max _{\theta \in \mathbb{R}}\left|\sum_{t=1}^{m} \cos (t \theta) a_{m, t} / t\right| .
$$

and that

$$
\begin{aligned}
\lim _{m \rightarrow+\infty} \frac{S_{x, m}^{A}\left(\mathrm{e}^{\mathrm{i} \theta_{m}^{\max }}\right)}{\sum_{t=1}^{m} a_{m, t} t t} & =\lim _{m \rightarrow+\infty} \frac{\alpha_{n} f_{m}\left(\theta_{m}^{\max }-\lambda_{n}\right)+\alpha_{n} \mathfrak{b}_{m}\left(\theta_{m}^{\max }-\lambda_{n}\right)+S_{x-v, m}^{A}\left(\mathrm{e}^{\mathrm{i} \theta_{m}^{\max }}\right)}{S_{\mathbb{1}, m}^{A}(1)} \\
& =\alpha_{n} \lim _{m \rightarrow+\infty} \frac{f_{m}\left(\theta_{m}^{\max }-\lambda_{n}\right)}{S_{\mathbb{1}, m}^{A}(1)}
\end{aligned}
$$

if the latter limit exists. Indeed, the sequences $\mathfrak{b}_{m}\left(\theta_{m}^{\max }-\lambda_{n}\right)$ and $S_{x-v, m}^{A}\left(\mathrm{e}^{\mathrm{i} \theta_{m}^{\max }}\right)$ are bounded due to (4.4) and $\omega \in B_{1}$, respectively.

Since $f_{m}\left(\theta_{m}^{\max }-\lambda_{n}\right) \leq S_{\mathbb{1}, m}^{A}(1)+|\mathfrak{b}|_{\infty}$, we see that $\limsup _{m \rightarrow \infty} \frac{f_{m}\left(\theta_{m}^{\max }-\lambda_{n}\right)}{S_{\mathbb{1}, m}^{A}(1)} \leq 1$. Thus, to prove (4.10) it is enough to show that

$$
\liminf _{m \rightarrow+\infty} \frac{f_{m}\left(\theta_{m}^{\max }-\lambda_{n}\right)}{S_{\mathbb{1}, m}^{A}(1)} \geq 1
$$

Since $C_{1}:=\sup _{m \in \mathbb{N}} \max _{\left|\theta-\lambda_{n}\right|<\gamma}\left|S_{x-v, m}\left(\mathrm{e}^{\mathrm{i} \theta}\right)\right|<\infty$, we see that for $\theta \in\left[\lambda_{n}-\gamma, \lambda_{n}+\gamma\right]$,

$$
\left|S_{x, m}^{A}\left(\mathrm{e}^{\mathrm{i} \theta}\right)-\alpha_{n} f_{m}\left(\theta-\lambda_{n}\right)\right| \leq C_{1}+|\mathfrak{b}|_{\infty}\left|\alpha_{n}\right| .
$$

Hence, for $\widetilde{\theta}_{m} \in\left\{\theta_{m, j}\right\}_{j=j_{m, n}}^{j_{m, n}^{\prime}}$ satisfying $\left|\mathrm{e}^{\mathrm{i} \widetilde{\theta}_{m}}-\mathrm{e}^{\mathrm{i} \lambda_{n}}\right|=\min _{j_{m, n} \leq j \leq j_{m, n}^{\prime}}\left|\mathrm{e}^{\mathrm{i} \widetilde{\theta}_{m, n}}-\mathrm{e}^{\mathrm{i} \lambda_{n}}\right|$, one has

$$
\left|\alpha_{n}\right| f_{m}\left(\theta_{m}^{\max }-\lambda_{n}\right) \geq\left|S_{x, m}^{A}\left(\mathrm{e}^{\mathrm{i} \theta_{m}^{\max }}\right)\right|-C_{1}-|\mathfrak{b}|_{\infty}\left|\alpha_{n}\right| \geq\left|S_{x, m}^{A}\left(\mathrm{e}^{\mathrm{i} \widetilde{\theta}_{m}}\right)\right|-C_{1}-|\mathfrak{b}|_{\infty}\left|\alpha_{n}\right|,
$$

where the definition of $\theta_{m}^{\max }$ was used to obtain the last inequality. Applying (4.21) once again, we see that

$$
\left|\alpha_{n}\right| f_{m}\left(\theta_{m}^{\max }-\lambda_{n}\right) \geq\left|\alpha_{n}\right| f_{m}\left(\widetilde{\theta}_{m}-\lambda_{n}\right)-2 C_{1}-2|\mathfrak{b}|_{\infty}\left|\alpha_{n}\right| .
$$


Therefore,

$$
\liminf _{m \rightarrow+\infty} \frac{f_{m}\left(\theta_{m}^{\max }-\lambda_{n}\right)}{S_{\mathbb{1}, m}^{A}(1)} \geq \liminf _{m \rightarrow+\infty} \frac{f_{m}\left(\widetilde{\theta}_{m}-\lambda_{n}\right)}{S_{\mathbb{1}, m}^{A}(1)} .
$$

From the monotonicity of $f_{m}$ on $[-\pi, 0]$ and $[0, \pi]$, one can see that

$$
f_{m}\left(2 \pi / J_{m}\right) \leq f_{m}\left(\widetilde{\theta}_{m}-\lambda_{n}\right) \leq f_{m}(0) \leq S_{\mathbb{1}, m}^{A}(1)+|\mathfrak{b}|_{\infty}
$$

This together with (4.8) and (4.22) implies (4.20) and completes the proofs of statement (4.10) and of the theorem.

\subsection{Proof of Proposition 4.3}

The following lemma can be obtained from (4.6) and elementary estimates.

Lemma 4.5. For the matrix 4.11), the functions $f_{m}$ of (4.2)- 4.4) can be taken as $f_{m}(\theta)=\max \left\{0,-\ln \left|1-\mathrm{e}^{-1 / m+\mathrm{i} \theta}\right|\right\}$. In this case, $|\mathfrak{b}|_{\infty}<\ln 2+\pi / 3+\mathrm{e}^{-1}<11 / 5$. Moreover,

$$
\left|S_{\mathbb{1}, m}^{A}\left(\mathrm{e}^{\mathrm{i} \theta}\right)\right| \geq-\ln \left|1-\mathrm{e}^{-1 / m+\mathrm{i} \theta}\right|-\mathrm{e}^{-1} \quad \text { for }|\theta| \leq \pi / 3 .
$$

Now, the proof of Proposition 4.3 consists of three steps.

Step 1. Let us show that

$$
\left|S_{\mathbb{1}, m}^{A}\left(\mathrm{e}^{\mathrm{i} \theta}\right)\right| \geq \frac{p+1}{2} \ln m-\frac{1}{2} \quad \text { for } \quad|\theta| \leq \frac{1}{m^{p+1 / 2}}, p=0,1, m \in \mathbb{N} .
$$

From $|\theta| \leq 1<\pi / 3$ and (4.23), one can see that it is enough to prove $\left|1-\mathrm{e}^{-1 / m+\mathrm{i} \theta}\right|^{2} \leq$ $1 / m^{p+1}$ or, equivalently, $4 \sin ^{2}(\theta / 2) \leq \mathrm{e}^{1 / m} / m^{p+1}+2-\left(\mathrm{e}^{1 / m}+\mathrm{e}^{-1 / m}\right)$. The latter follows for $|\theta| \leq 1 / m^{p+1 / 2}$ from $|\sin (\theta / 2)| \leq|\theta| / 2$ and the fact that

$$
x^{p+1} \mathrm{e}^{x}+2-\left(\mathrm{e}^{x}+\mathrm{e}^{-x}\right) \geq x^{2 p+1} \text { for } x \geq 0 \text { and } p=0,1 .
$$

Step 2. Let $H_{m}=\frac{1}{2} \ln m-\frac{1}{2}$. Then (4.24) with $p=0$ implies (3.8) for $J_{m} \geq 2 \pi \sqrt{m}$, and so also (3.6). Taking $h_{m}$ and $h_{m}^{\prime}$ defined by (4.12), one ensures (4.1).

Step 3. Let us put $\theta_{m}=2 \pi / \widetilde{J}_{m}$ with $\widetilde{J}_{m} \in \mathbb{N}$ such that

$$
\left|S_{\mathbb{1}, m}^{A}\left(\mathrm{e}^{\mathrm{i} \theta_{m}}\right)\right| \geq \ln m-\frac{1}{2} .
$$

Applying (4.24) with $p=1$, we see that (4.25) holds whenever $\widetilde{J}_{m} \geq m^{3 / 2}$. This means that for $J_{m} \geq 2 \pi m^{3 / 2}$, we obtain with the use of (4.6) that

$$
1 \geq \frac{\left|S_{\mathbb{1}, m}^{A}\left(\mathrm{e}^{\mathrm{i} 2 \pi / J_{m}}\right)\right|}{S_{\mathbb{1}, m}^{A}(1)} \geq \frac{\ln m-1 / 2}{-\ln \left(1-\mathrm{e}^{-1 / m}\right)+1 / \mathrm{e}} \text { and, in turn, } \lim _{m \rightarrow \infty} \frac{\left|S_{\mathbb{1}, m}^{A}\left(\mathrm{e}^{\mathrm{i} 2 \pi / J_{m}}\right)\right|}{S_{\mathbb{1}, m}^{A}(1)}=1 .
$$

It follows from $J_{m} \rightarrow+\infty$ and (4.6) that $\lim _{m \rightarrow \infty} \frac{S_{1, m}^{A}\left(\mathrm{e}^{\mathrm{i} 2 \pi / J_{m}}\right)}{\left|S_{1, m}^{A}\left(\mathrm{e}^{\mathrm{i} 2 \pi / J_{m}}\right)\right|}=1$. This and (4.26) complete the proof of (4.8) and of the proposition. 


\section{Discussion and additional remarks}

\subsection{Relaxation of signal and noise assumptions}

While the case of decaying useful part $(y(t))_{t=1}^{\infty}$ of the signal was addressed in Statistics in connection with significance of related statistical hypotheses [3], we are not aware of studies of consistency of estimators for such models. Models with decaying $(y(t))_{t=1}^{\infty}$ appears, e.g., in the analysis of chirp-type signals [19, 1] (after inversion of the time direction) or in the passing from idealized conservative models of various tomography techniques to models with attenuated waves [8].

Signal processing intuition suggests that if the decaying deterministic signal (i.e., $y(t) \rightarrow 0$ as $t \rightarrow \infty)$ is corrupted by a random noise $\epsilon_{t}$ of 'constant strength', then 'the signal-to-noise ratio' goes to 0 as $t \rightarrow \infty$ and it is difficult to expect that standard estimators for such a signal remain asymptotically consistent.

On the other side, an estimator as a function of $\{x(t)\}_{t=1}^{m}$ may cancel to some extend the influence of the random noise sequence $\left\{\epsilon_{t}\right\}_{t=1}^{m}$. Theorem 4.2 shows that this cancellation can be strong enough to recover parameters of $y(\cdot)$ if the decay of $y(\cdot)$ is not very fast. Indeed, consider the model $x(t)=\sum_{n=1}^{N} \alpha_{n} \frac{\mathrm{e}^{-\mathrm{i} \lambda} \lambda_{n}}{t^{\xi}}+\epsilon_{t}$, where $\lambda_{n}$ and $\alpha_{n}$ are as before, $\epsilon_{t}$ are i.i.d. symmetric with finite variances, and $\xi \in \mathbb{R}_{+}$is the rate of power decay of the deterministic terms $y(t)=\sum_{n=1}^{N} \alpha_{n} \frac{\mathrm{e}^{-\mathrm{i} \lambda_{n} t}}{t^{\xi}}$. Then the rescaled signal $\widetilde{x}(t)=t^{\xi} x(t)$ is of form (1.1). If $\xi<1 / 2$, the rescaled noise sequence $\widetilde{\epsilon}_{t}:=t^{\xi} \epsilon_{t}$ satisfies (N3), and so, Theorem 4.2 is applicable.

This observation and the sequence of assumptions (N1)-(N3) naturally lead to the following question.

Problem 1. To what extend is it possible to relax the assumptions on the random variables $\epsilon_{t}$ and the model for deterministic part $y(\cdot)$ of the signal such that the recovery of the parameters of $y(\cdot)$ by a certain asymptotically consistent procedure remains possible.

Another way to relax assumptions on the model for $y(\cdot)$ is to allow the number $N$ of frequencies to be (countably) infinite. The assumption that $N$ is finite may be non-reasonable for some of applications. For example, many of models of mechanical, acoustical, and electro-magnetic resonators lead to infinite number of eigen-frequencies [8, 16]. Generic excitation generates a signal containing all of them. While only some finite number of frequencies have large enough amplitudes to be interesting, the infinite sum of the rest still contribute to the signal. This contribution cannot be treated as independent random noise terms $\epsilon_{t}$. That is why it is reasonable to include signals with possibly infinite $N$ into consideration, but to recover only frequencies with comparatively large amplitudes $\left|\alpha_{n}\right|$.

It is not difficult to see that the consistent localization statement $\left(\lim _{m \rightarrow \infty} \widehat{L}_{m}=\operatorname{supp} \mu\right.$ a.s.) of Theorem 3.2 remains valid in the case when $N=\infty$ under the additional assumptions that $\sum_{n=1}^{\infty}\left|\alpha_{n}\right|<\infty$ and every frequency $z_{n}$ is an isolated point of the set $\left\{z_{n}\right\}_{n=1}^{\infty}$ of all frequencies. 


\subsection{Probability estimates for accurate localization}

The study of the precision of the above estimators requires knowledge of probability bounds on distribution tails of $\mathcal{L}^{\infty}(\mathbb{T})$-norms of random trigonometric polynomials $S_{\epsilon, m}^{A}\left(\mathrm{e}^{\mathrm{i} \theta}\right)$ constructed by the noise sequence $\left(\epsilon_{t}\right)_{t=1}^{m}$. To illustrate this, consider probability estimates on accuracy of the procedure of localization of $\operatorname{supp} \mu=\left\{z_{n}\right\}_{n=1}^{N}$ by the union $\widehat{L}_{m}$ of superlevel arcs (see Section 3.2).

In the sequel, we assume the truncated power summation matrix is defined by (4.11), and that $H_{m}, h_{m}, J_{m}$, and $h_{m}^{\prime}$ are chosen in accordance with (3.6)-(3.8) and (4.1). For $\theta_{1,2} \in \mathbb{R}$, let us denote by $\operatorname{dist}_{\mathbb{T}}\left(\mathrm{e}^{\mathrm{i} \theta_{1}}, \mathrm{e}^{\mathrm{i} \theta_{2}}\right)$ (by $\operatorname{dist}_{\mathbb{T}}\left(\mathrm{e}^{\mathrm{i} \theta_{1}}, G\right)$ ) the 'circular' distances between $\mathrm{e}^{\mathrm{i} \theta_{1}}$ and $\mathrm{e}^{\mathrm{i} \theta_{2}}$ (resp., $\mathrm{e}^{\mathrm{i} \theta_{1}}$ and the set $G \subset \mathbb{T}$ ). More precisely, $\operatorname{dist}_{\mathbb{T}}\left(\mathrm{e}^{\mathrm{i} \theta_{1}}, \mathrm{e}^{\mathrm{i} \theta_{2}}\right):=\min _{k \in \mathbb{N}}\left|\theta_{2}-\theta_{1}-2 \pi k\right|, \operatorname{dist}_{\mathbb{T}}\left(\mathrm{e}^{\mathrm{i} \theta_{1}}, G\right):=\inf _{z \in G} \operatorname{dist}_{\mathbb{T}}\left(\mathrm{e}^{\mathrm{i} \theta_{1}}, z\right)$. Recall that $\|\mu\|=\sum_{n=1}^{\infty}\left|\alpha_{n}\right|=\int_{\mathbb{T}}|\mathrm{d} \mu|$.

Proposition 5.1. (i) Assume that $\mathrm{e}^{\mathrm{i} \lambda_{n}}$ is the only point of $\operatorname{supp} \mu$ in $\operatorname{Arc}\left(\lambda_{n}-\Delta, \lambda_{n}+\Delta\right)$ and that $\Delta>2 \pi / J_{m}$. Then

$\operatorname{Pr}\left(\mathrm{e}^{\mathrm{i} \lambda_{n}} \notin \widehat{L}_{m}\right) \leq \operatorname{Pr}\left(\left\|S_{\epsilon, m}^{A}\right\|_{\infty} \geq\left|\alpha_{n}\right| H_{m}-h_{m}-\left(\|\mu\|-\left|\alpha_{n}\right|\right)\left(f_{m}\left(\Delta-2 \pi / J_{m}\right)+|\mathfrak{b}|_{\infty}\right)\right)$.

(ii) Assume that $z \in \mathbb{T}$ is such that $\operatorname{dist}_{\mathbb{T}}(z, \operatorname{supp} \mu) \geq \Delta \geq 2 \pi / J_{m}$. Then

$$
\operatorname{Pr}\left(z \in L_{m}\right) \leq \operatorname{Pr}\left(\left\|S_{\epsilon, m}^{A}\right\|_{\infty} \geq h_{m}-\|\mu\|\left(f_{m}\left(\Delta-\pi / J_{m}\right)+|\mathfrak{b}|_{\infty}\right)\right) .
$$

Statements (i) and (ii) also hold if one changes $L_{m}$ and $h_{m}$ to $L_{m}^{\prime}$ and $h_{m}^{\prime}$, respectively.

Proof. (i) Put $v(t)=\alpha_{n} \mathrm{e}^{-\mathrm{i} \lambda_{n} t}$. Since $\Delta>2 \pi / J_{m}$, there exist $\theta_{i}=2 \pi l_{i} / J_{m}, i=1,2$, such that $l_{i} \in \mathbb{Z}, l_{2}=l_{1}+1$, and $\mathrm{e}^{\mathrm{i} \lambda_{n}} \in \operatorname{Arc}\left[\theta_{1}, \theta_{2}\right]$.

Suppose $\mathrm{e}^{\mathrm{i} \lambda_{n}} \notin \widehat{L}_{m}$. Then at least one of the two inequalities $\left|S_{x, m}^{A}\left(\mathrm{e}^{\mathrm{i} \theta_{i}}\right)\right|<h_{m}$, $i=1,2$, holds. Let us fix such $\theta_{i}$ and note that the corresponding inequality implies

$$
\begin{aligned}
\left|S_{\epsilon, m}^{A}\left(\mathrm{e}^{\mathrm{i} \theta_{i}}\right)\right| \geq\left|S_{v, m}^{A}\left(\mathrm{e}^{\mathrm{i} \theta_{i}}\right)\right|-h_{m}-\left|S_{y-v, m}^{A}\left(\mathrm{e}^{\mathrm{i} \theta_{i}}\right)\right| \geq \\
\quad \geq\left|\alpha_{n}\right| H_{m}-h_{m}-\left(\|\mu\|-\alpha_{n}\right)\left(f_{m}\left(\Delta-2 \pi / J_{m}\right)+|\mathfrak{b}|_{\infty}\right) .
\end{aligned}
$$

To show the last estimate, it is enough to notice that $\left\{\mathrm{e}^{\mathrm{i} \lambda_{j}}\right\}_{j \neq n} \cap \operatorname{Arc}\left[\theta_{i}-\Delta+2 \pi / J_{m}, \theta_{i}+\right.$ $\left.\Delta-2 \pi / J_{m}\right]=\varnothing$ and that (4.2)-(4.4) imply

$$
\left|S_{y-v, m}^{A}\left(\mathrm{e}^{\mathrm{i} \theta_{i}}\right)\right| \leq \sum_{j \neq n}\left|\alpha_{j}\right|\left(f_{m}\left(\theta_{i}-\lambda_{j}\right)+|\mathfrak{b}|_{\infty}\right) \leq \sum_{j \neq n}\left(f_{m}\left(\Delta-2 \pi / J_{m}\right)+|\mathfrak{b}|_{\infty}\right) \sum_{j \neq n}\left|\alpha_{j}\right| .
$$

(ii) Assume that $z \in \operatorname{Arc}\left[\theta_{1}, \theta_{2}\right]$, where $\theta_{1,2}=2 \pi l_{1,2} / J_{m}$ with $l_{1,2} \in \mathbb{Z}$ and $l_{2}=l_{1}+1$. Then $z \in \widehat{L}_{m}$ if and only if $\left|S_{x, m}^{A}\left(\mathrm{e}^{\mathrm{i} \theta_{i}}\right)\right| \geq h_{m}$ for $i=1,2$. Since $\operatorname{dist}_{\mathbb{T}}\left(z, \mathrm{e}^{\mathrm{i} \theta_{i}}\right) \leq \pi / J_{m}$ for at least one of $i=1,2$, we see that, for this $i,\left|S_{\epsilon, m}^{A}\left(\mathrm{e}^{\mathrm{i} \theta_{i}}\right)\right| \geq h_{m}-\left|S_{y, m}^{A}\left(\mathrm{e}^{\mathrm{i} \theta_{i}}\right)\right| \geq$ $h_{m}-\|\mu\|\left(f_{m}\left(\Delta-\pi / J_{m}\right)+|\mathfrak{b}|_{\infty}\right)$. 
The estimates on the distribution tails of random polynomials rely on concentration inequalities and, up to our knowledge, are available only under the subgaussian or subexponential assumptions on $\epsilon_{t}$.

Let us consider the subgaussian case. Recall that a real random variable $\xi$ is called subgaussian with the scaling factor $b \geq 0$ if $\mathbb{E}\left(e^{t \xi}\right) \leq \mathrm{e}^{b^{2} t^{2} / 2}$ for all $t \in \mathbb{R}$.

Lemma 5.2. Consider a complex trigonometric polynomial $q(\theta)=\sum_{k=0}^{n} a_{k} \xi_{k} \mathrm{e}^{\mathrm{i} k \theta}$, where $a_{k} \in \mathbb{C}$ and where $\xi_{k}$ are i.i.d. complex random variables such that $\operatorname{Re} \xi_{k}$ and $\operatorname{Im} \xi_{k}$ are independent and subgaussian with the scaling factors $b_{1}$ and $b_{2}$, respectively. Assume that $b_{1,2}$ are chosen such that $b_{1} \geq b_{2} \geq 0$ and $b_{1}>0$. Put $r:=b_{1}^{2} \sum_{k=0}^{n}\left|a_{k}\right|^{2}$. Then, $\operatorname{Pr}\left(\|q\|_{\infty} \geq C\right) \leq(n+1) 2 \pi \mathrm{e}^{3 / 2}\left(\frac{C^{2}}{2 r}-1\right) \exp \left(-\frac{C^{2}}{4 r}\right)$ whenever $C>2 \sqrt{2 r}$.

This lemma can be obtained by modification of the subgaussian arguments of Kahane [10, Section 6.2] (which are based on the Salem-Zygmund use of Bernstein inequalities [35]).

From now on, suppose that the noise satisfies the following assumption:

(Nsg) $\epsilon_{t}$ are i.i.d. complex random variables such that for a certain $\varphi \in[-\pi, \pi)$ the random variables $\operatorname{Re}\left(\mathrm{e}^{\mathrm{i} \varphi} \epsilon_{k}\right)$ and $\operatorname{Im}\left(\mathrm{e}^{\mathrm{i} \varphi} \epsilon_{k}\right)$ are independent and subgaussian with the scaling factors $b_{1}$ and $b_{2}$, respectively, chosen such that $b_{1} \geq b_{2} \geq 0$ and $b_{1}>0$.

Lemma 5.2 implies that under this assumption

$$
\operatorname{Pr}\left(\left\|S_{\epsilon, m}^{A}\left(e^{\mathrm{i} \theta}\right)\right\|_{\infty} \geq C\right) \leq \frac{\pi \mathrm{e}^{3 / 2}}{b_{1}^{2} \sum_{t=1}^{m} a_{m, t}^{2} / t^{2}} m C^{2} \exp \left(-\frac{3}{2 \pi^{2} b_{1}^{2}} C^{2}\right)
$$

for $C \geq 2 \pi b_{1} / \sqrt{3}$ (we have used here the facts that $a_{m, t} \leq 1$ and $\sum_{t=1}^{m} a_{m, t}^{2} / t^{2}<\pi^{2} / 6$ ).

Let $O_{\Delta}(\operatorname{supp} \mu)$ be the open in the topology of $\mathbb{T}$ neighborhood of supp $\mu$ defined by

$$
O_{\Delta}(\operatorname{supp} \mu):=\left\{z \in \mathbb{T}: \operatorname{dist}_{\mathbb{T}}(z, \operatorname{supp} \mu)<\Delta\right\}, \quad \Delta>0 .
$$

By $\mathcal{A}(\widetilde{\alpha}, \Delta)$ we denote the set of all points $z$ of $\operatorname{supp} \mu$ that bear a complex mass $\alpha$ with $|\alpha|>\widetilde{\alpha}$ and satisfy $\operatorname{dist}_{\mathbb{T}}(z, \operatorname{supp} \mu \backslash\{z\}) \geq \Delta$.

The above bound (5.3) on the distribution tail of $\left\|S_{\epsilon, m}^{A}\left(\mathrm{e}^{\mathrm{i} \theta}\right)\right\|_{\infty}$ can be combined with Proposition 5.1 to obtain, for instance, the following result.

Corollary 5.3. Let $m \geq 3, h_{m}=\ln ^{1-\delta} m$ with $\delta \in(0,1 / 2)$, and $J_{m}=\left\lceil 2 \pi m^{1 / 2}\right\rceil$. Let us consider, for $\widetilde{\alpha}>0$ and $\Delta \in(0, \pi / 3)$, the two following events

$$
B_{m}^{\mathcal{A}}(\widetilde{\alpha}, \Delta):=\left\{\mathcal{A}(\widetilde{\alpha}, \Delta) \subset \widehat{L}_{m}\right\}, \quad B_{m}^{\text {dist }}(\Delta):=\left\{\widehat{L}_{m} \subset O_{\Delta}(\operatorname{supp} \mu)\right\} .
$$

Then,

$$
\begin{gathered}
\limsup _{m \rightarrow \infty} \frac{\ln \left(1-P\left(B_{m}^{\mathcal{A}}(\widetilde{\alpha}, \Delta)\right)\right)}{\ln ^{2} m} \leq-\frac{3 \widetilde{\alpha}^{2}}{8 \pi^{2} b_{1}^{2}}, \\
\limsup _{m \rightarrow \infty} \frac{\ln \left(1-P\left(B_{m}^{\text {dist }}(\Delta)\right)\right)}{\ln ^{2-2 \delta} m} \leq-\frac{3}{8 \pi^{2} b_{1}^{2}} .
\end{gathered}
$$




\section{References}

[1] Abbott, B.P., et al., 2016. Observation of gravitational waves from a binary black hole merger. Physical review letters, 116(6), p.061102.

[2] Arciero, M., 2007. A limit theorem for Szegö polynomials with respect to convolution of point masses with the Fejér kernel. Journal of mathematical analysis and applications, 327(2), pp.908-918.

[3] Brillinger, D.R., 1987. Fitting cosines: some procedures and some physical examples (pp. 75-100). In "Applied Probability, Stochastic Processes and Sampling Theory", Vol. 1 (ed. I.B. MacNeill and G.J. Umphrey), Springer Science \& Business Media.

[4] Cuzick, J. and Lai, T.L., 1980. On random Fourier series. Transactions of the American Mathematical Society, 261(1), pp.53-80.

[5] Filbir, F., Mhaskar, H.N. and Prestin, J., 2012. On the problem of parameter estimation in exponential sums. Constructive Approximation, 35(3), pp.323-343.

[6] Fuchs, J.J., 1988. Estimating the number of sinusoids in additive white noise. Acoustics, Speech and Signal Processing, IEEE Transactions on, 36(12), pp.18461853.

[7] Hardy, G.H., 2000. Divergent series. American Mathematical Soc.

[8] Holman, B. and Kunyansky, L., 2015. Gradual time reversal in thermo-and photoacoustic tomography within a resonant cavity. Inverse Problems, 31(3), p.035008.

[9] Jones, W.B., Njåstad, O. and Saff, E.B., 1990. Szegö polynomials associated with Wiener-Levinson filters. Journal of Computational and Applied Mathematics, 32(3), pp.387-406.

[10] Kahane, J.P., 1993. Some random series of functions. Cambridge University Press.

[11] Kavalieris, L. and Hannan, E.J., 1994. Determining the number of terms in a trigonometric regression. Journal of time series analysis, 15(6), pp.613-625.

[12] Kliger, M. and Francos, J.M., 2013. Strongly Consistent Model Order Selection for Estimating 2-D Sinusoids in Colored Noise. Information Theory, IEEE Transactions on, 59(7), pp.4408-4422.

[13] Kritchman, S. and Nadler, B., 2009. Non-parametric detection of the number of signals: Hypothesis testing and random matrix theory. Signal Processing, IEEE Transactions on, 57(10), pp.3930-3941.

[14] Kunis, S., Peter, T., Römer, T. and von der Ohe, U., 2016. A multivariate generalization of Prony's method. Linear Algebra and its Applications, 490, pp.31-47. 
[15] Kunis, S., Möller, H.M. and von der Ohe, U., 2016. Prony's method on the sphere. arXiv preprint arXiv:1603.02020.

[16] Kunyansky, L., Holman, B. and Cox, B.T., 2013. Photoacoustic tomography in a rectangular reflecting cavity. Inverse Problems, 29(12), p.125010.

[17] Markel, J.D. and Gray, A.J., 2013. Linear prediction of speech. Springer Science \& Business Media.

[18] Mhaskar, H.N. and Prestin, J., 2000. On the detection of singularities of a periodic function. Advances in Computational Mathematics, 12(2-3), pp.95-131.

[19] Morvidone, M. and Torrésani, B., 2003. Time scale approach for chirp detection. International Journal of Wavelets, Multiresolution and Information Processing, 1(01), pp.19-49.

[20] Nadler, B. and Kontorovich, L., 2011. Model selection for sinusoids in noise: Statistical analysis and a new penalty term. Signal Processing, IEEE Transactions on, 59(4), pp.1333-1345.

[21] Njåstad, O. and Waadeland, H., 1997. Asymptotic properties of zeros of orthogonal rational functions. Journal of computational and applied mathematics, 77(1), pp.255-275.

[22] Pan, K., 1996. A refined Wiener-Levinson method in frequency analysis. SIAM Journal on Mathematical Analysis, 27(5), pp.1448-1453.

[23] Pan, K. and Saff, E.B., 1992. Asymptotics for zeros of Szegö polynomials associated with trigonometric polynomial signals. Journal of Approximation Theory, 71(3), pp.239-251.

[24] Papy, J.M., De Lathauwer, L. and Van Huffel, S., 2007. A shift invariance-based order-selection technique for exponential data modelling. IEEE signal processing letters, 14(7), pp.473-476

[25] Paulraj, A., Roy, R., Kailath, T., 1985. Estimation of signal parameters via rotational invariance techniques-ESPRIT (pp. 83-89), In Nineteeth Asilomar Conference on Circuits, Systems and Computers, IEEE.

[26] Peter, T. and Plonka, G., 2013. A generalized Prony method for reconstruction of sparse sums of eigenfunctions of linear operators. Inverse Problems, 29(2), 025001 (21 pp.).

[27] Plonka, G. and Tasche, M., 2014. Prony methods for recovery of structured functions. GAMM-Mitteilungen, 37(2), pp.239-258.

[28] Potts, D. and Tasche, M., 2010. Parameter estimation for exponential sums by approximate Prony method. Signal Processing, 90(5), pp.1631-1642. 
[29] Potts, D. and Tasche, M., 2013. Parameter estimation for nonincreasing exponential sums by Prony-like methods. Linear Algebra and its Applications, 439(4), pp.1024-1039.

[30] Potts, D. and Tasche, M., 2013. Parameter estimation for multivariate exponential sums. Electronic Transactions on Numerical Analysis, 40, pp.204-224.

[31] Pillai, S.U., 2012. Array signal processing. Springer Science \& Business Media.

[32] Quinn, B.G. and Hannan, E.J., 2001. The estimation and tracking of frequency. Cambridge University Press.

[33] Rockafellar, R.T. and Wets, R.J.B., 2009. Variational analysis. Springer Science \& Business Media.

[34] Roy, R. and Kailath, T., 1989. ESPRIT-estimation of signal parameters via rotational invariance techniques. Acoustics, Speech and Signal Processing, IEEE Transactions on, 37(7), pp.984-995.

[35] Salem, R. and Zygmund, A., 1954. Some properties of trigonometric series whose terms have random signs. Acta Mathematica, 91(1), pp.245-301.

[36] Stoica, P. and Moses, R.L., 2005. Spectral analysis of signals. Upper Saddle River, NJ: Pearson/Prentice Hall.

[37] Stoica, P., Moses, R.L., Friedlander, B. and Söderström, T., 1989. Maximum likelihood estimation of the parameters of multiple sinusoids from noisy measurements. Acoustics, Speech and Signal Processing, IEEE Transactions on, 37(3), pp.378392.

[38] Zhou, W.X. and Sornette, D., 2002. Statistical significance of periodicity and logperiodicity with heavy-tailed correlated noise. International Journal of Modern Physics C, 13(2), pp.137-169.

Illya M. Karabash, Institute of Applied Mathematics and Mechanics of NAS of Ukraine, Dobrovolskogo st. 1, Slovyans'k 84100, Ukraine, and Humboldt Research Fellow at the University of Bonn, Endenicher Allee 60, D-53115 Bonn, Germany.

E-mail: i.m.karabash@gmail.com

Jürgen Prestin, Institut für Mathematik, Universität zu Lübeck, Ratzeburger Allee 160, D-23562 Lübeck, Germany.

E-mail: prestin@math.uni-luebeck.de 